\title{
Retrieval of cloud spherical albedo from top-of-atmosphere reflectance measurements performed at a single observation angle
}

\author{
A. Kokhanovsky ${ }^{1}$, B. Mayer ${ }^{2}$, W. von Hoyningen-Huene ${ }^{1}$, S. Schmidt ${ }^{3}$, and P. Pilewskie ${ }^{3}$ \\ ${ }^{1}$ Institute of Environmental Physics, University of Bremen, Otto Hahn Allee 1, 28334 Bremen, Germany \\ ${ }^{2}$ Institute of Atmospheric Physics, Deutsches Zentrum für Luft- und Raumfahrt (DLR), Oberpfaffenhofen, 82234 Wessling, \\ Germany \\ ${ }^{3}$ Laboratory for Atmospheric and Space Physics, University of Colorado, Boulder, CO 80309-0311, USA
}

Received: 25 November 2005 - Published in Atmos. Chem. Phys. Discuss.: 31 March 2006

Revised: 22 May 2007 - Accepted: 4 July 2007 - Published: 11 July 2007

\begin{abstract}
The paper is aimed to the derivation of the simple analytical relationship between the cloud spherical albedo and the cloud reflection function in the visible. The relationship obtained can be used for the retrieval of the spherical albedo from backscattered solar light measurements performed by radiometers on geostationary and polar orbiting satellites. The example of the application of the technique to MODIS data is shown.
\end{abstract}

\section{Introduction}

The determination of the spherical albedo $r$ from satellite observations (see, e.g., Diner et al., 2005) is a difficult task because the value of $r$ is an integral of the measured cloud reflection function with respect to angular variables (e.g., the solar and satellite observation angles, the relative azimuth). One way to derive the spherical albedo is to determine the cloud optical thickness and the effective droplet/ice crystal size from spectral top-of-atmosphere reflectance measurements (e.g., using a look-up-table technique). Subsequently, this information can be used for the calculation of $r$.

Interestingly, there is an alternative possibility of the direct determination of the spherical cloud albedo from single reflection function measurements for the special case of optically thick cloudiness. The proposed technique requires no a priori information on the size of droplets and the cloud optical thickness. The accuracy of the technique can be increased if information on the cloud thermodynamic state (e.g., from thermal infrared measurements) is known. The method proposed is based on the asymptotic radiative transfer equation solution valid for optically thick cloud layers (van de Hulst, 1980; Kokhanovsky, 2006).

Correspondence to: A. Kokhanovsky

(alexk@iup.physik.uni-bremen.de)

\section{The determination of cloud spherical albedo}

The reflection function $R(\xi, \eta, \varphi)$ of an optically thick nonabsorbing cloud over black surface can be presented in the following form in the visible (van de Hulst, 1980; Kokhanovsky, 2006):

$R(\xi, \eta, \varphi)=R_{\infty}(\xi, \eta, \varphi)-t K(\xi) K(\eta)$,

where $t$ is the diffuse cloud transmittance under diffuse illumination conditions, $R_{\infty}(\xi, \eta, \varphi)$ is the reflection function of a semi-infinite cloud, $K(\xi)$ is the escape function, $\xi$ is the cosine of the solar angle, $\eta$ is the cosine of the observation angle, and $\varphi$ is the relative azimuth. This equation has a high accuracy for clouds with the optical thickness larger than 10 (Kokhanovsky, 2006). Taking into account that $t=1-r$ due to the energy conservation law, we obtain from Eq. (1):

$r=1-\frac{R_{\infty}(\xi, \eta, \varphi)-R(\xi, \eta, \varphi)}{K(\xi) K(\eta)}$.

This analytical result can be used to determine the cloud spherical albedo from the reflection function $R(\xi, \eta, \varphi)$ measurements at a single viewing geometry in the visible. No additional information on cloud microphysical characteristics and cloud extent is needed for the retrieval procedure. However, the information on the cloud thermodynamic state is of importance. This is due to the fact that the function $R_{\infty}(\xi, \eta, \varphi)$ actually depends on the shape of scatterers. The dependence of $R_{\infty}(\xi, \eta, \varphi)$ on the size of particles can be neglected as demonstrated by Kokhanovsky et al. (2003). This means that the pre-calculated look-up-table of $R_{\infty}(\xi, \eta, \varphi)$ can be used in combination with Eq. (2) for the direct determination of the water cloud spherical albedo from satellite observations. Yet another look-up-table must be constructed for the specific case of ice clouds.

Published by Copernicus Publications on behalf of the European Geosciences Union. 


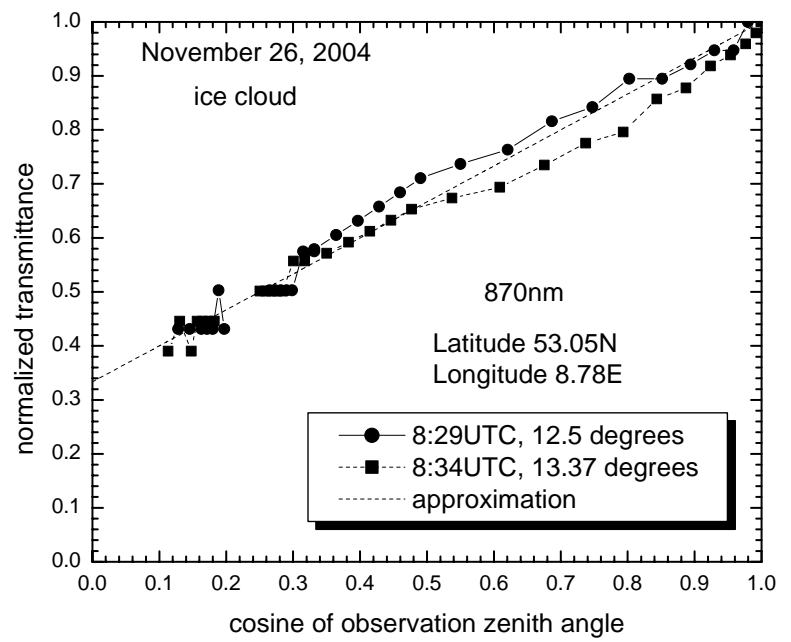

Fig. 1. The cloud transmittance divided by its value at the zenith. Measurements have been performed under crystalline optically thick precipitating cloud conditions in Bremen (northern Germany) on 24 November 2004. Only results for the principal plane measurements at the wavelength $870 \mathrm{~nm}$ are shown. Solar zenith angles were 12.5 (08:29 UTC) and 13.37 (08:34 UTC) degrees, respectively. The field of view was $1.2^{\circ}$.

The escape function $K(\xi)$ in Eq. (2) is almost insensitive to the shape/size of scatterers (Kokhanovsky, 2003) and can be approximated as follows:

$K(\xi)=\frac{3}{7}(1+2 \xi)$.

Such a simple form is due to the fact that this function describes the angular distribution of photons escaping from a semi-infinite nonabsorbing media with sources located deep inside the medium. Therefore, multiple light scattering washes out almost all features characteristic for a single scattering law (at least for $\xi \geq 0.2$, which is a standard case for most cloud remote sensing problems). To check the theoretical result as shown in Eq. (3), we have performed measurements of the angular distribution of light transmitted by a cloud in the principal plane using Automatic Sun Tracking Photometer CE318. The details of the instrument are given at http://www.cimel.fr.

The cloud was composed of precipitating ice crystals. Results of measurements are given in Fig. 1. Indeed, we see that measurements nicely follow the cosine law as predicted by Eq. (3). The broken line corresponds to the value of the escape function given by Eq. (3) divided by $K(1)=9 / 7$.

Substituting Eq. (3) in Eq. (2), we get:

$r=1-\frac{49\left(R_{\infty}(\xi, \eta, \varphi)-R(\xi, \eta, \varphi)\right)}{9(1+2 \xi)(1+2 \eta)}$,

where $R(\xi, \eta, \varphi)$ must be obtained from measurements and $R_{\infty}(\xi, \eta, \varphi)$ is given either in a correspondent look-up-table

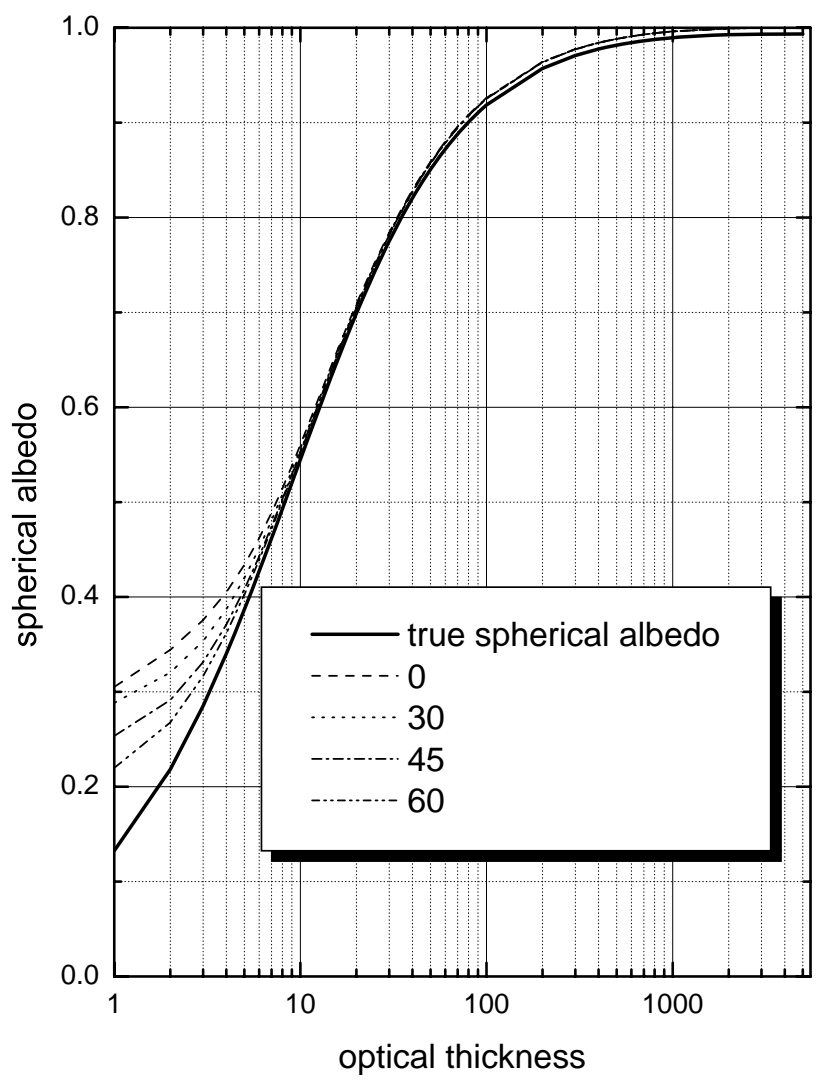

Fig. 2. Spherical albedo calculated using exact radiative transfer code (solid line) and also obtained using approximate Eq. (2) for several solar zenith angles $\left(0,30,45\right.$, and $\left.60^{\circ}\right)$ as function of cloud optical thickness.

or by an approximation (Kokhanovsky, 2006). In particular, the following approximation for $R_{\infty}$ at $\eta=1$ in the case of water clouds can be used (Kokhanovsky, 2006):

$R_{\infty}=\frac{0.37+1.94 \xi}{1+\xi}+\frac{p(\pi-\arccos \xi)}{4(1+\xi)}$.

Here, $p(\pi-\arccos \xi)$ is the cloud phase function.

The accuracy of Eq. (4) is shown in Fig. 2. The spherical albedo for solar zenith angles $0,30,45$, and $60^{\circ}$ was obtained using the numerical solution of the radiative transfer equation in the framework of libradtran package (Kylling and Mayer, 2005) for different cloud optical thicknesses $\tau$ in the range 1-5000 (solid line in Fig. 2). Calculations were performed at the wavelength $650 \mathrm{~nm}$ for the gamma droplet size distribution with the effective radius $6 \mu \mathrm{m}$ and the coefficient of variance equal to 0.37 . Effects of light absorption were neglected. Broken lines give calculations of $r$ using Eq. (4) with $R_{\infty}(\xi, \eta, \varphi)$ and $R(\xi, \eta, \varphi)$ obtained from libradtran. It follows that there is dependence of $r$ on the solar zenith angle at $\tau \leq 10$. This means that Eq. (2) is not suitable for the case of thin clouds. However, for thicker clouds solid and broken lines almost coincide (see Fig. 2). This confirms the 
applicability of the technique for optically thick clouds. The absolute value of the error is smaller than $10 \%$ at $\tau \geq 6$. The error is below 3\% at $\tau \geq 10$ for all considered solar zenith angles and nadir observations. We have found that the method can be recommended for the values of the spherical albedo larger than 0.5 (or even 0.4 , depending on the accuracy required).

Equation (4) is less accurate, if not the look-up-table (LUT) but rather the analytical result (see Eq. 5) for the reflection function of a semi-infinite cloud is used in calculations.

We find that the following analytical result at $\eta=1$ combining Eqs. (4) and (5) and neglecting $p$ :

$r=1-\frac{2.01+10.56 \xi-5.44(1+\xi) R(\xi, \eta, \varphi)}{3(1+\xi)(1+2 \xi)}$.

This formula can be used for rapid and yet accurate estimations of the cloud spherical albedo from the reflection function measurements at the nadir observation conditions for a black underlying surface. Actually, the extension of the theory to the case of underlying Lambertian surface with the albedo $A$ is straightforward. Then the term $A t^{2} K(\xi) K(\eta)(1-A r)^{-1}$ must be added to Eq. (1) (Kokhanovsky, 2006) and Eq. (2), therefore, transforms to

$r=(c(1-A)-b)(c(1-A)-b A)^{-1}$,

where $b=R_{\infty}(\xi, \eta, \varphi)-R(\xi, \eta, \varphi)$ and $c=K(\xi) K(\eta)$.

\section{The application of the technique to airborne measure- ments}

We applied Eq. (6) for relating spectral radiance and irradiance data acquired simultaneously onboard the NASA high altitude ER-2 aircraft during the Cirrus Regional Study of Tropical Anvils and Cirrus Layers - Florida Area Cirrus Experiment (CRYSTAL-FACE) in July 2002. Solar spectral irradiance was measured by the Solar Spectral Flux Radiometer (SSFR, Pilewskie et al., 2003), radiance by the MODIS Airborne Simulator (MAS; King et al., 2004).

The spatial resolution of MAS at cloud top across and along the flight track was $20 \mathrm{~m}$, the swath about $13 \mathrm{~km}$ wide. In contrast to MAS directional radiance measurements, the SSFR measured up- and downward irradiance, which is the cosine-weighted integral of radiance in the lower and upper hemisphere, respectively. The measured cloud albedo (upward normalized by downward irradiance) is representative for the whole underlying cloud field; the area where $50 \%$ of the measured albedo (irradiance) originate is called footprint of the SSFR. In this case, the footprint is about $13 \mathrm{~km}$ in diameter. Consequently, about 650 of the $20 \mathrm{~m}$ MAS pixels contribute to the measured irradiance.

For testing the radiance-irradiance conversion, we chose a flight leg on 9 July 2002, from UTC 18:35-18:55, entirely above the Gulf of Mexico where a thick cloud (single layer) was probed. From the MAS level 1B data, we

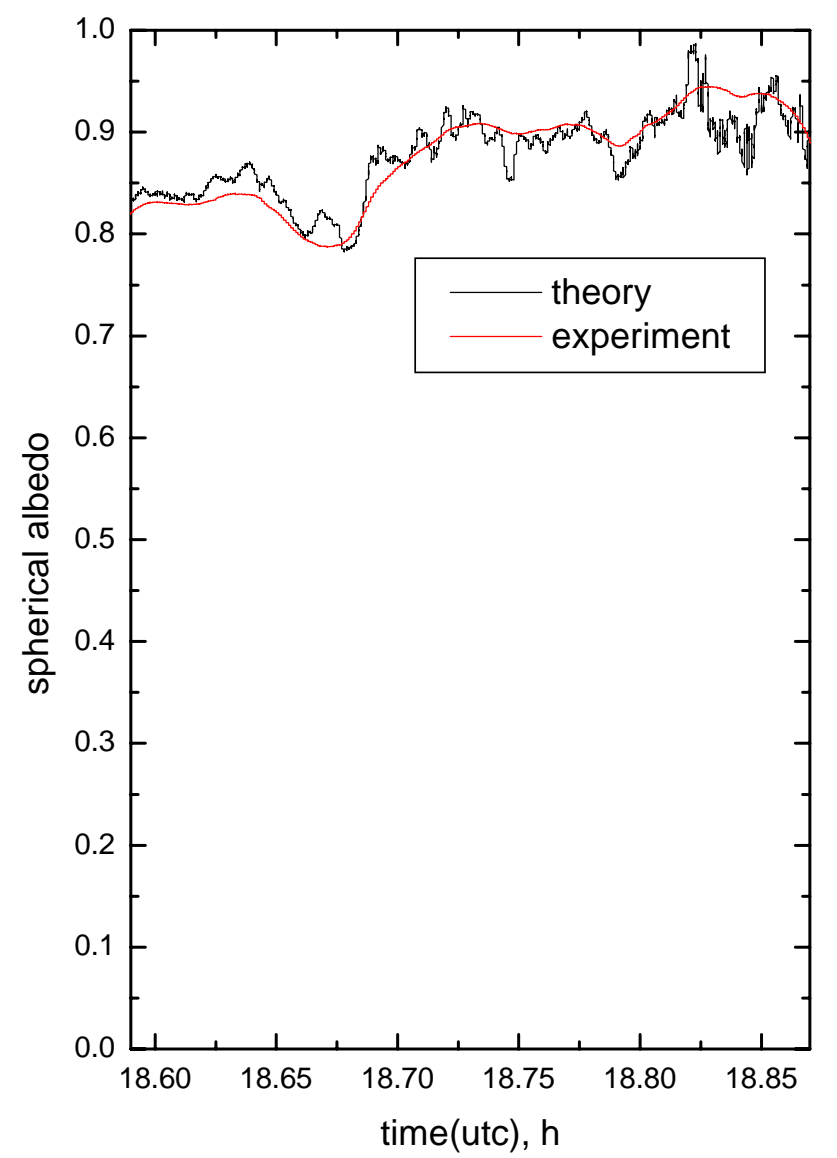

Fig. 3. Measured and calculated albedo at $555 \mathrm{~nm}$ above a thick cloud measured on 9 July 2002, in the Gulf of Mexico.

extracted the calibrated radiance from band $2(528-570 \mathrm{~nm})$ along the flight track, as well as sensor and solar geometry, which we used as input to Eq. (6) and compared the result to SSFR measured irradiance at $555 \mathrm{~nm}$. The results are shown in Fig. 3 with measurements in red, and calculations in black. There is a remarkable agreement between measured and calculated albedo; differences occur for example at UTC $=18.65 \mathrm{~h}$ and $18.84 \mathrm{~h}$; also, the calculated albedo shows far more variability than the measured counterpart. Both effects are caused by the different area over which the radiance and irradiance observations are made: While the radiance measurements originate from $20 \mathrm{~m}$ MAS pixels, the SSFR albedo is a hemispherical quantity, with $50 \%$ originating within a circle of $13 \mathrm{~km}$ diameter. When averaging radiance-derived albedo across the entire MAS swath within the SSFR footprint (not done for simplicity), there should be even closer agreement. Future tests may include other solar wavelengths and clouds with different degree of inhomogeneity. Expected result is that with increasing cloud inhomogeneity, a larger cloud area needs to be considered for reproducing irradiance from a radiance field. 

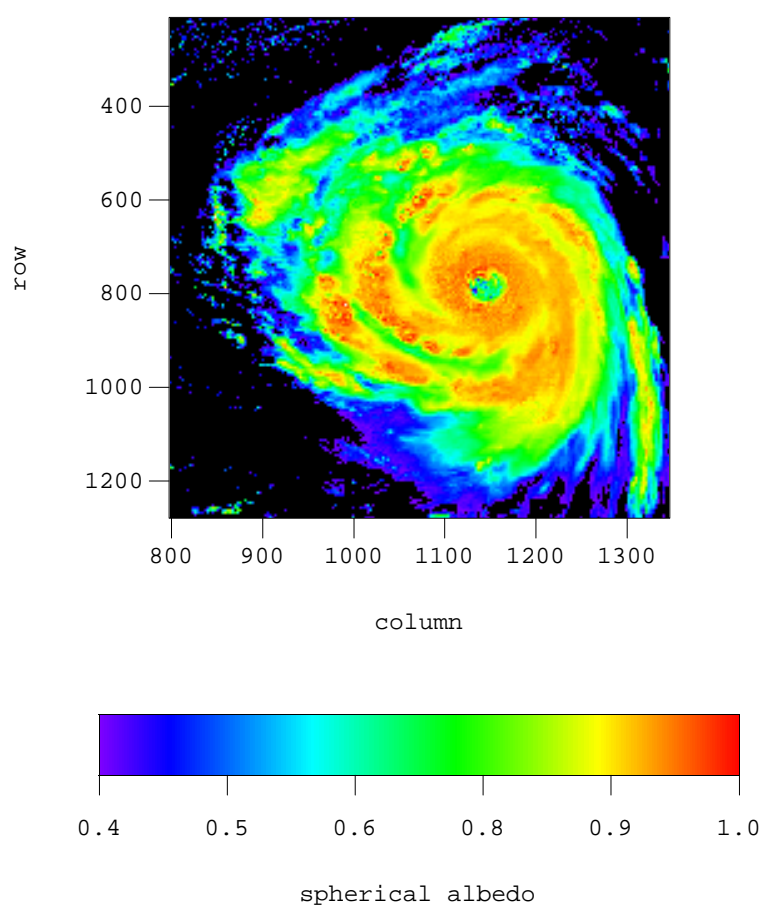

Fig. 4. The spatial distribution of the spherical albedo.

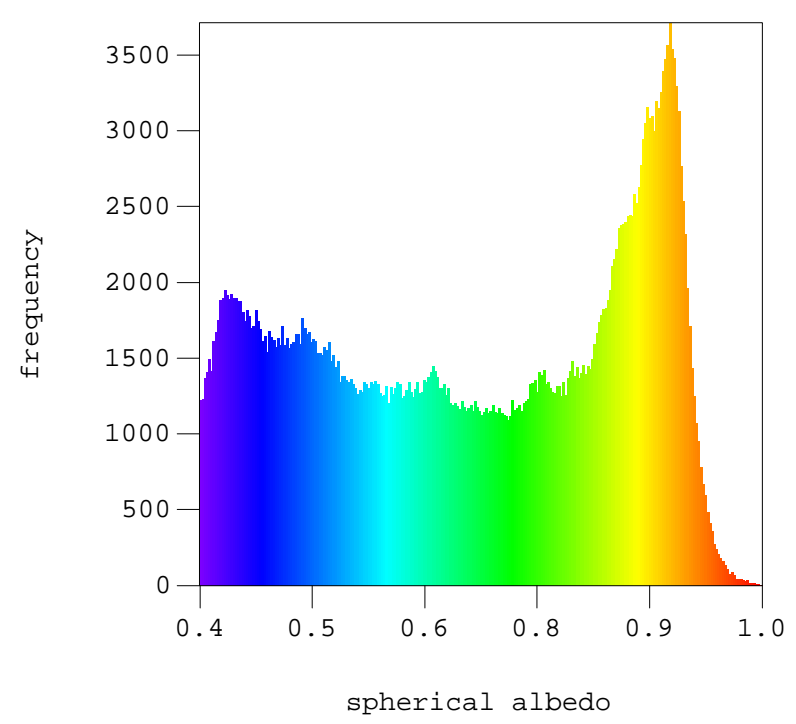

Fig. 5. The spherical albedo histogram.

\section{The application of the technique to satellite data}

We applied the technique described above for studies of the spherical albedo of Hurricane Kathrina using top-ofatmosphere reflectance measurements of MODIS on Terra satellite. Hurricane Katrina has originated as a Tropical De- pression on 23 August 2005 at $\left(23.2^{\circ} \mathrm{N}, 75.5^{\circ} \mathrm{W}\right)$. Hurricane has propagated initially to north-west, then to west and finally to north and then to north-east (after landfall in New Orlean). The retrieved spherical albedo map for 28 August 2005 (17:00 UTC) is shown in Fig. 4. MODIS measurements at $645 \mathrm{~nm}$ with the spatial resolution $1 \mathrm{~km}$ were used for the creation of this map. Therefore, the spatial scale on the map shown in Fig. 4 corresponds to $1 \mathrm{~km}$. The cloud spherical albedo retrieval is based on Eq. (2) with $R_{\infty}(\xi, \eta, \varphi)$ found for a special case of crystalline clouds as described by Kokhanovsky (2005). This is due to the fact that tops of hurricanes are covered by ice clouds.

We see that Katrina occupies the huge area of approximately $400 \times 800 \mathrm{~km}^{2}$. At this date Katrina was classified as Hurricane category 5 with winds $175 \mathrm{mph}$ and a minimum pressure of 902 mbar. The next day Kathrina was downgraded to Category 4 hurricane and made a landfall in New Orlean.

It follows that hurricane, as one might expect, is characterized by quite large values of the spherical albedo (larger than 0.9 for the central part of a hurricane). The spherical albedo in an eye is somewhat smaller (around 0.7-0.8). It means that an eye is occupied by quite thick clouds in the case considered. The histogram of cloud spherical albedo is shown in Fig. 4. Colors in Figs. 4 and 5 have the same meaning. So we conclude that the spherical albedo of a hurricane is larger than 0.6 for most of $1 \times 1 \mathrm{~km}^{2}$ pixels studied. Blue color corresponds to relatively thin clouds on the periphery of the cloud system. Here broken cloud conditions not accounted in the theory described above can influence retrievals considerably.

\section{Conclusions}

We propose here a simple analytical result (see Eq. 4), which can be used for the determination of cloud spherical albedo in the visible from top-of-atmosphere reflectance measurements (at a single viewing geometry). The error is smaller than 3 percent at cloud optical thicknesses larger than 10 . Therefore, the error is smaller than the correspondent calibration errors of optical instruments currently orbiting the planet. The procedure described above does not require a priori knowledge of the cloud optical thickness and the effective radius of scatterers and valid for the case of optically thick clouds. The spherical albedo map and also the frequency of occurrence of $r$ for Hurricane Katrina were studied. It was found that the most frequent value of spherical albedo is around 0.9 for Category 5 Hurricane Katrina with the largest fraction of clouds with albedos in the range 0.6-0.95. Our findings confirm that hurricanes are strong reflectors of visible radiation back to space. This could have important climatic effects taking into account the increased frequency of strong hurricanes in recent years. 


\section{References}

Diner, D. J., Braswell, B. H., Davies, R., Gobron, N., Hu, J., Jin, Y., Kahn, R. A., Knyazikhin, Y., Loeb, N., Muller, J.-P., Nolin, A. W., Pinty, B., Schaaf, C. B., Seiz, G., and Stroeve, J.: The value of multiangle measurements for retrieving structurally and radiatively consistent properties of clouds, aerosols, and surfaces, Rem. Sens. Environ., 97, 495-518, 2005.

King, M. D., S. Platnick, P. Yang, G. T. Arnold, M. A. Gray, J. C. Riedi, S. A. Ackerman, and K. N. Liou: Remote sensing of liquid water and ice cloud optical thickness and effective radius in the Arctic: Application of airborne multispectral MAS data. J. Atmos. Oceanic Technol., 21, 857-875, 2004.

Kokhanovsky, A. A., Rozanov, V. V., Zege, E. P., Bovensmann, H., and Burrows, J. P.: A semianalytical cloud retrieval algorithm using backscattered radiation in $0.4-2.4 \mu \mathrm{m}$ spectral band, J. Geophys. Res., 108, 4008, doi:10.1029/2001JD001543, 2003.
Kokhanovsky, A. A.: Reflection of light from particulate media with irregularly shaped particles, J. Quant. Spectr. Rad. Transfer, 96, 1-10, 2005.

Kokhanovsky, A. A.: Cloud optics, Berlin, Springer, 2006.

Kylling, A. and Mayer, B.: Technical note: The libRadtransoftware package for radiative transfer calculations - description and examples of use, Atmos. Chem. Phys., 5, 1855-1877, 2005, http://www.atmos-chem-phys.net/5/1855/2005/.

Pilewskie, P., Pommier, J., Bergstrom, R., Gore, W., Howard, S., M. Rabbette, Schmid, B. Hobbs, P. V., and Tsay, S. C.: Solar spectral radiative forcing during the Southern African Regional Science Initiative, J. Geophys. Res., 108(D13), 8486, doi:10.1029/2002JD002411, 2003.

van de Hulst, H. C.: Multiple light scattering, N.Y., Academic Press, 1980. 